\title{
A Novel Method for Secure Outsourced in Cloud through KAE Authentication
}

\author{
V N Rajavarman, SrinivasaRao Madala, T.Venkata Satya Vivek
}

\begin{abstract}
Development of The cloud computing technology establishes a huge paradigm shift in the functional process of the IT industry. Since this new handling technology needs clients to believe in their useful information to reasoning providers, there have been enhancing security and comfort problems on shortened details. A few strategies using quality based security (ABE) have been prescribed for access administration of abbreviated subtle elements in thinking processing; be that as it may, the greater part of them experience from resoluteness in actualizing complex accessibility administration rules. The significant ones are capable of summing up with each other to develop vital variables and it could develop as traditional self-contained key, however covering the substantial number of keys being accumulated. In different terms, the key proprietor can discharge consistent size of separate key with proceedings of data for flexible alternatives of data that is composed of content set residing within the distributed storage space; however more significant and secured data documents which stay outside the set however kept classified. The lightly loaded overall key set could be the appropriate global pass to users which could be associated with a smart card with extremely confined safeguarded storage room. In our experiments evaluation official security examinations will provide to official cloud data storage events.
\end{abstract}

Index Terms: Attribute based encryption, Cloud Computing, secure Hashing, Scalable and reliable data encryption and decryption.

\section{INTROUDCTION}

Cloud computing is a replica for authorize extensive system access to share the configurable PC assets. Multilied figure and accumulate choices furnish customer and organizations with different ability to load and system their data in vistor data offices [1]. It relies on upon talking about of sources to expert reasonability and monetary frameworks of extent, like an application (like the force network) above a framework. At the base of cloud preparing is the more extensive thought of consolidated offices and disseminated administrations.
Revised Manuscript Received on October 12, 2019

Dr.V.N.Rajavarman, Professor and Deputy Dean, Computer Science ,Dr.M.G.R Educational and Research Institute University, Chennai

Mr.M.Srinivasarao, Assistant Professor, Computer Science and Engineering Department of PACE Institute of Technology and Sciences, Ongole.

T.Venkata Satya Vivek, Department of Computer Science \& Engineering from Vishnu Institute of Technology, Bhimavaram, India,

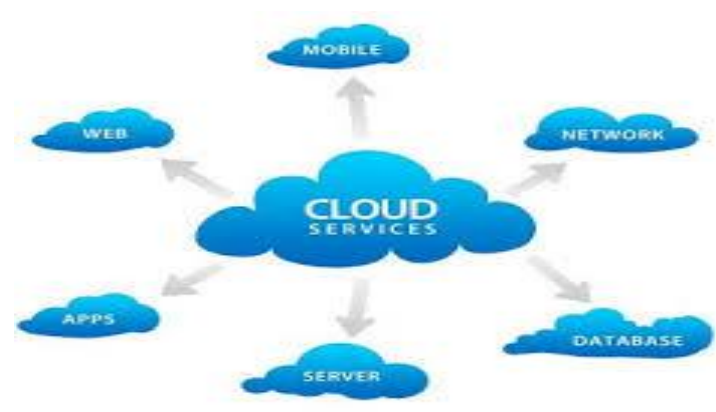

Figure 1: Resource monitoring encompasses could computing [15].

The above figure signifies three major solutions provided by the cloud computing service to the thinking support and the cloud computing service also includes distributed management roles. Thinking handling utilized three types of services such as PASS, SAAS and Facility As Service as well to monitor the storage space information. Therefore information handling and preservation of information significantly comprises of customer activities and presentations of data which is used in motivation program [2]. Some of the companies possess the ACPs program which controls the client activity and helps them to decide about the information sharing. Therefore the ACPs are often demonstrated as the characteristics of the clients, for the most part known as distinguishing proof components, utilizing availability administration dialects, for example, XACML. Such a methodology, for the most section it is recognized as assets based accessibility controllability (ABAC) which encourages condensed openness to the administration that is pivotal for affirmative data security and support.

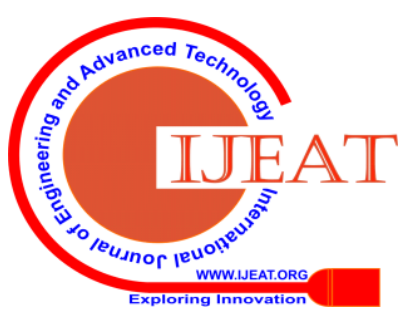




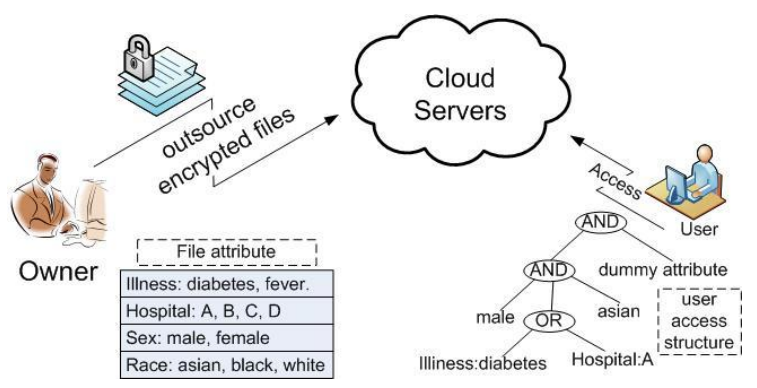

Figure 2: encryption based on data characteristics used for outsourcing [2].

The cipher text massages could only be decrypted by the companies containing specific characteristics which is controlled and monitored by Attribute-based Security (ABE) [3][4]. It is suitable for accessibility management as the computers files demonstrates methods. Many other companies can be presented for the idea of decryption trough the cipher published text. The ABE utilizes the client's features as the recognition process and the client's set of features are protected through decrypting details. There are some limitation that could be observed among modern $\mathrm{ABE}$ systems which could be used for resource-limited devices as the system uses combined features of clients and hence the number of combined features which are required to be decrypted through cipher published written text that needs more complex strategy. There are some ABE strategy which could be implemented to resolve the issue whenever client's detailed need to be used.

Alice is a general user of the cloud technology and she put her picture into the drop box which she doesn't want to disclose to anyone. Because of distinct data security problems Alice could not rely directly on the Dropbox's support assurance module due to data security issues, so she encrypts the pictures and utilizes own vital variables before posting on public platform. On a given day Bob, Alice's friend told her to suppress everyone in the picture where Bob was present in the picture. Alice utilizes the editor's option available in Dropbox and shared the rights to Bob to manage the data in the picture. Alice securely delivered the vital keys to Bob which enable him to edit any information in the picture. There are two unbalanced system available in accordance with normal security system parameters.

Alice could secure all information and data with a single security solution and avoids involving any security key. Alice on the other hand could ensure the information through applying exceptional vital components with complex key variables.

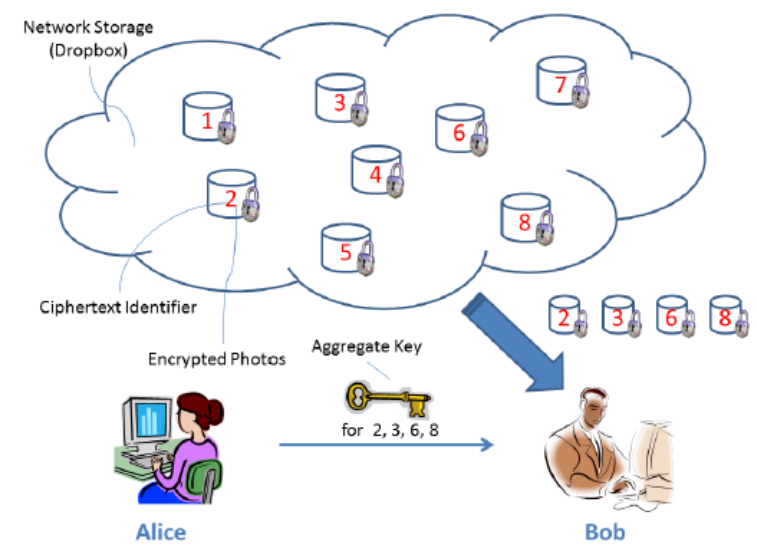

Figure 3: Alice shares the information with Bob through the identifiers $2,3,6$ and 8 by sending him only one set of key [16].

As mentioned in the above figure 3 , surely, the initial plan is lacking security since all data were supplied to Bob which enforces the process to the next technique that is some real problems arises while executing the security process. The vital components used in this case are the combination of millions of common pictures. Therefore a secured sequence of action is needed while moving these vital keys and Therefore moving these secured and vital components needs more storage space [6]. The expenses and muddling connected with typically enhance with the mixed bag of the unscrambling essential variables to be conveyed. In a nutshell, it is exceptionally gigantic and extravagant to do that. Encoding essential components likewise partner two tastes - special key or uneven key. Using the secret key coding process, when Alice looks for the information to be initiate from a third break, Alice desires to share the coding or the vital keys and decrypt it which is not a suitable process. By complexity, security clue and decoding clue are distinctive in plan day glow clue security. Therefore using the unlocking hints for the encryption process creates more flexibility for our projects. For example, in business projects, each specialist could transfer encoded information to the control room server without the data of the organization's mystery key which belongs to the expert.

The finest way out from the problem is Alice gathers all data with unresolved hints and decodes the information with it [8]. The complex and combined clues to be sent through protected support and vital set of key, therefore, minimum clue measurement is suitable in this case. For instance, we could not uncertain about gigantic stockpiling for unscrambling imperative elements in the benefit need appliances as approach cells, astute charge cards or Wi-Fi pointer axis.

Especially, these key essential variables are typically spared in the carefully designed capacity, which is generally spending. The adjacent investigation activity focuses on minimizing the cooperation particulars.

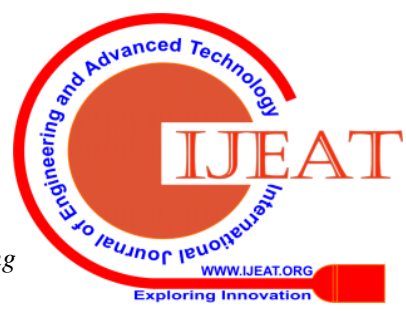


The rest of the discussion would be arranged in the following mentioned proportion such as: Section II helps us to create an overview of the previous works whereas in Section III the Traditional approach in case of application with security considerations would be discussed; it also elaborates the effectiveness of the data demonstration as well as the outline of the planned tactic. The section IV focuses on the cloud data safety component as well as computational processes like implementation and performance evaluation. Section V encloses the concluding portion of the cloud security proceedings.

\section{RELATED WORK}

Around there we clarify the method of project, we evaluation the idea of property based assurance (ABE), and give a brief finish of the ASBE system by Bobba et al. After that, we assess present accessibility control systems relying upon ABE. Characteristic Based Security The idea of ABE was initially given by Sahai and Wealthy rich waters [4] as another route for indeterminate personality based assurance. The essential disadvantage of the technique in [4] is that, it confine semantics does not have impressibility. A few ventures followed in the anecdotal perform to attempt to alter the impressibility issue. In the ABE technique, figure instant messages are not legitimately secured to one specific customer as in conventional gathering key cryptography. Maybe, both figure instant messages and clients' unscrambling key components are connected with an arrangement of capacities or a method over capacities. A customer has the capacity decode a figure composed content just if there is an arrange between his unscrambling key and the figure composed content. ABE strategies are ordered into key-arrangement characteristic based assurance (KP-ABE) and figure content approach trait based security (CP-ABE), in view of how capacities and system are connected with figure instant messages and clients' unscrambling key components. In any case, essential CP-ABE routines (e.g., [5]) are a long way from enough to returning up accessibility control in present day organization environment, which require vital flexibility and effectiveness in determining proposals and overseeing customer capacities [4]. In a CP-ABE procedure, unscrambling key components just bolster customer capacities that are sorted out judiciously as one and only set, so customers can just utilize every single conceivable blend of capacities in one and only set propelled in their key components to get together with proposals. To settle this issue, Bobba et al. [4] gave figure content strategy trait set-based insurance (CPASBE or ASBE for short). ASBE is a broadened method for $\mathrm{CP}-\mathrm{ABE}$ which sets up customer capacities into a recursive set structure. The accompanying is an illustration of a key system of points of interest 2, which is subtle elements of the recursive set structure:

\{ CS:Dept, Role: Huge - Student,

\{Course ID: 101, Role: TA, \}

\{Course ID: 525, Role: Grand- $\square$ Student\}\}

The above sample speaks to a key system allotted to a graduate understudy college understudy in CS branch of a remarkable, who is the TA for course 101 and has approved in course 525. It can be seen that the same work can be allocated a few ideas, e.g., the work "Part" is doled out quality "TA" and "Graduate Student" in diverse areas. This work gives ASBE more adaptable and adaptable in supporting numerous genuine conditions. In this sample, the graduate understudy college understudy having such an individual key ought not have the capacity to consolidate the work "Part: TA" with "Coursed 525" to accessibility course elements of different understudies who join course 525 . Such a work can't be utilized with the interesting CP-ABE necessities. The conventional technique to ensured delicate subtle elements abbreviated to third exercises is to store appropriately secured points of interest on web servers, while the decoding critical variables are presented to acknowledged customers just. Be that as it may, there are a few downsides about this simple arrangement. As a matter of first importance, such an answer needs a proficient key control methodology to disseminate unscrambling key components to acknowledged customers, which has been confirmed to be exceptionally confounded. Next, this system does not have versatility and adaptability; as the extensive variety of acknowledged customers gets to be enormous, the arrangement won't be proficient any longer. In situation a some time ago valid customer should be denied, suitable points of interest must be re-encoded and new key components must be allocated to introduce true customers once more. To wrap things up, points of interest organization proprietors should be on the web record-breaking in order to ensured or re-encode subtle elements and circulate essential elements to acknowledge customers. This methodology permits a subtle elements proprietor to dispense a large portion of the computational expense to thinking web servers. The utilization of KP-ABE gives fine-grained accessibility control greatly. Every PC data document is appropriately secured with a formed points of interest encryption key ( ), which is thus legitimately secured by a gathering key relating to an arrangement of capacities in $\mathrm{KP}-\mathrm{ABE}$, which is made by accessibility system.

\section{KEY AGGREGATION ENCRYPTION}

We would be contributing to the overall meaning and framework of the key of total security. Secondly we would elaborate the usage of KAC within the program with respect to cognitive structure of the storage space.

Structure: There are five polynomial-time techniques are incorporated into the key total security procedure which would be elaborated in below section.

The SETUP procedure would be done by the data manager and generates professional secret pair of keys applying KEY GEN. The data would be secured through the encryption process by a professional who significantly choose the content classification which would be connected with the secured data [8][9] The data manager deploys an expert to develop the overall secret key for the data through days of research and application. The developed vital variables would be deployed secretly
Published By:

Blue Eyes Intelligence Engineering \& Sciences Publication 
through secured massages or applications. Therefore any user who is containing the total secret key can decrypt the data classification with ease. .

Shared Encrypted Data: The primary focus of the discussion is to explain the idea of information in context with cloud storage space through using the KAC as shown in figure 3. For instance, let's take the case of Alice who want to discuss the information series such as $\mathrm{m} 1, \mathrm{~m} 2$....mn. Alice would be executing the KEY GEN $\left(1^{\lambda} ; \mathrm{n}\right)$ which create the public secret key that is pk:msk which are the parameters that is the program parameter of PARAM and the public key that is pk would be published whereas the secret key namely msk kept secured with Alice. She and any other person who containing the key composition would be protecting the mi through $\mathrm{Ci}=$ encryption. Later the encoded information set would be surrender to the server. Alice's co-workers who are containing the PARAM and pk are capable of upgrading the information set by accessing the server. Whenever Alice willing to share the information set such as KS with her friend Bob, she could remove the S securely to assure the data security. Alice could share the information KS through email as it is a continuous zonal clue. As Alice approved the accessibility of the information to Bob, the information could be downloaded by using the total key [10]. Therefore, Bob could download the $\mathrm{Ci}$ from the secured email for each specified i $2 \mathrm{~S}$. The key KS enable Bob to decode every unit $\mathrm{Ci}$ through decoding (KS; $\mathrm{S}$; $;$; $\mathrm{Ci}$ ).

\section{IMPLEMENTATION OF KAC}

There are two primary acquisitions such as $\mathrm{T}$ and $\mathrm{TG}$ which are within repeated group: $£ \times £ \rightarrow £_{T}$ is the outline containing the below mentioned characteristics:

Bilinear: $\forall_{g 1, g 2} \in £, a, b \in \phi, \hat{e}(g, g)=\hat{e}(g 1, g 2)^{a b}$

Non-degenerate: There would be some $g \in £, \hat{e}(g, g) \neq 1$ which denotes all the functional parameters would be operated smoothly and $g$ is the bilinear team in the above mentioned scenario.

\subsection{Construction}

The essence of the primary plan was adopted from the collusion-resistant security plan as suggested and conveyed. The important factor in the security plan is the constant size of the key; each clue contains the capacity to decode cipher text messages which are attached with a specific set [8]. There is a need for new outline development corresponding to the decoding criteria specified for total key.

Structure: we significantly developed a bilinear team G which is associated with initial imperative $\mathrm{p}$ that is $2^{\lambda} \leq p \leq 2^{\lambda+1}$ and $\quad$ generator $g \in £$ and $\alpha \in_{R} \varnothing_{p}$ .evaluate the bilinear team $g_{i}=g^{\alpha^{i}} \in \mathcal{f}$ for $i=1, \ldots ., n, n+2, \ldots ., 2 n$ whereas the output parameters are set as param $=\left(g, g 1, \ldots . ., g_{n}, g_{n+2}, \ldots . ., g_{2 n}\right)$ It could be noticed that the each cipher massage set is associate with a integer set such as $i=1, \ldots ., n, n+2, \ldots ., 2 n$.

Key Gen: Choose $\gamma \in_{R} \notin_{p}$ for yielding master key and public key that is $\left(p k=v=g^{\gamma}, m s k=\lambda\right)$.

Encrypt: Let's take an Instance if a message structure contains $\quad m \in £_{T}$ alongside the index $i \in\{1,2,3, \ldots \ldots n\}$ which arbitrarily choose $t \in_{R} \phi_{p}$ and it would calculate the cipher text set $e=\left(g^{t},\left(v g_{i}\right)^{t}, m \cdot \hat{e}(g 1, g m)^{t}\right)$.

Decrypt $\left(K_{s}, S, i, e=(c 1, c 2, c 3)\right)$ : If $i \notin S$ output is the $\lambda$ or else $m=c_{3} \cdot \hat{e}\left(K_{s} \cdot \prod_{j \in s, j \neq i} g_{n+1-j+i}, c_{1}\right) / \hat{e}\left(\prod_{j \in S} g_{n+1-j}, c_{3}\right)$

\subsection{Performance}

^e(g1; gn) would be calculated initially which associated with program parameter to generate a secured process. It could be observed that the during the decryption process, there is a need of two pair of keys where only one has the total key structure [12]. Therefore it could inferred that the total key needs only one join calculation during the decryption process. The modern usage of compelling application is to withstand the pointer hub..

2.2. System Process

The "attraction" of obtaining consistent scope from the aggregate key set and steady magnitude of the composed content in the meantime originates from the direct size framework parameter.

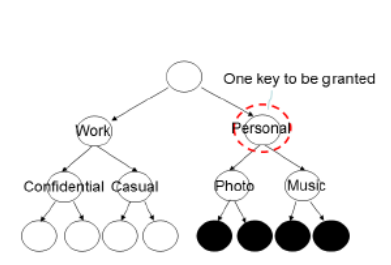

(a)

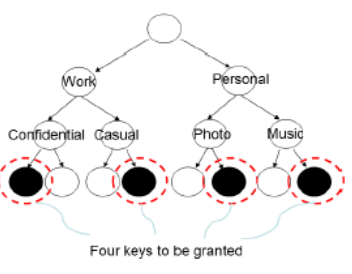

(b)

Figure 4: Compact key sometimes not possible in case of a fixed hierarchy [7].

The storage space would be surely decreased in the process and it would be an adjustment between two storage spaces. The overall program structure could be stored into closer by non-private data storage spaces.. The set of programs could be recalled as necessary and not all information sets are required for all events. The parameters associated with the program set would be created by trusted individuals, which differentiates between users and keeps the initial contact with the user's framework. The user needs to trust a parameter generator which could dispose any used parameter set. The availability of the parameters could be controlled by the encryption process.

\section{PERFORMANCE EVALUATION}

For a large assessment, we analyze the territory facts of tree-headquartered key set mission methodology. This has been used in association with the whole Sub tree association. It is used as partner resolution for the conveyed defense problem taking into account the Subset-cover

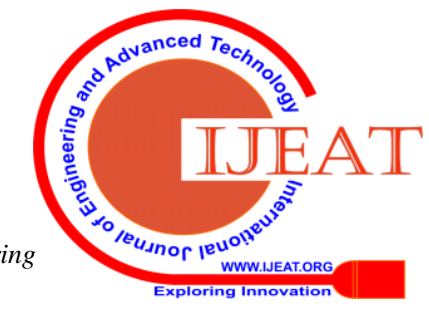


structure [13]. This structure uses a collection clever key constitution and it is observed with a whole double key bush of the dimension h (equals to three in determine 4), and it also can support as much as $2 \mathrm{~h}$ to control composed content classes and picked a section which is used as an confirmed agent.

In an textbook scenario as stated in Figure 5(a) and hence a agent could be supplied to the accessibility of the $2 \mathrm{hs}$ sessions that is controlling the only one key, whereas hs is stated as size of a sub tree (e.g., hs $=2$ as mentioned in Figure 5(a)). Therefore it is significant to decode cipher text messages associated with set of sessions and hence the agent has to possess huge number of significant factors which represented in the figure 5(b). Therefore, it is a serious concerned about na which is a part of various key structure assigned to the hierarchical key strategy.

We could consider that there are as many as $2 \mathrm{~h}$ cipher text sessions in the decryption process and the agent are eligible to access part of the issue associated with the session.. If $\mathrm{r}=0$, then the na should be tend to 0 which means, there is no availability to any of the classes and for instance if $r=100 \%$, the na would be 1 in this case, which shows the key ownership of the main the structure enables the user to get the accessibility to the $2 \mathrm{~h}$ sessions. Hence, anyone can assume that na would be growing with $\mathrm{r}$, which might decrease later [8]. If we choose the percentage of $r=10 \% ; 20 \% ; \ldots \ldots ; 90 \%$ and consecutively, and choose the section randomly to develop a distinct "delegation pattern" for multiple agents. A combination of sessions such as 104 sessions could be produced for each mixture of $h$ and $r$ and the resulting key set na is common over unique allocations.

\section{EXPERIMENTAL SETUP}

Our systems permit the strain facet $\mathrm{F}$ to be an adjustable parameter which is equivalent to the $\mathrm{O}(\mathrm{n})$-sized program parameter. Therefore, the safety procedure could be finished in nonstop time period and the decryption can be done in $\mathrm{O}(\mathrm{jSj})$ team duplications as it consisting of 2 pairing features. The $S$ could be mentioned as the set of cipher text periods which could be decrypted in a position with the support of the total key structure and $\mathrm{jSj}$ $\mathrm{n}$ [11]. It is estimated that the key removal looks for $\mathrm{O}(\mathrm{jSj})$ workforce growth which appears inevitable. Therefore it could be validated by means of research outcomes, we do not must set an extraordinarily first-rate $\mathrm{n}$ to have superior strain than the sapling-founded technique. Realize that category multiplication is an extraordinarily quick function.

Table1: Data handling with the total key structure in context with time effectiveness

\begin{tabular}{|l|l|}
\hline Depth of Key & Time Productivity \\
\hline 1 & 0.04965 \\
\hline 2 & 0.05974 \\
\hline 3 & 0.07052 \\
\hline 4 & 0.08572 \\
\hline
\end{tabular}

\begin{tabular}{|l|l|}
\hline 5 & 0.09760 \\
\hline
\end{tabular}

We could authenticate the empirically of our research is genuine. We could connect vital KAC program in $\mathrm{C}$ with the match-competition that is the Cryptography (PBC) Library8 releases 0.4.18. The key which is provided would be smaller in size that is $1 \mathrm{G}$ and cipher text only comprises of two G and a single GT components. We used the (symmetric) of groupings of Type-A (super singular) shapes as it is labeled in the $\mathrm{PBC}$ collection which provides the biggest performance amongst of all shapes and the Type-A shapes will not offer any of the quickest reflection for the team components.

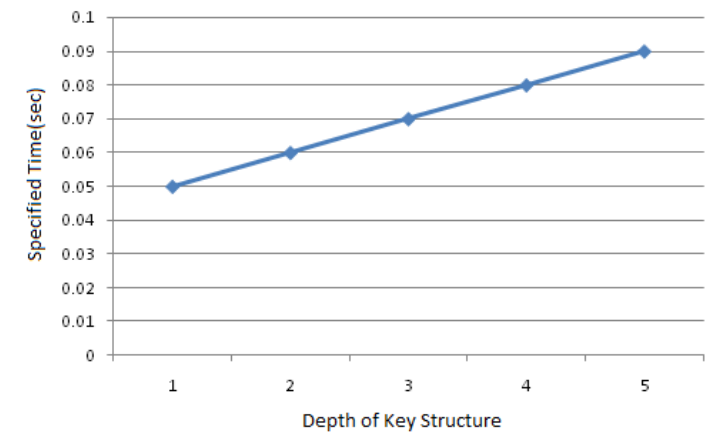

Figure 5: Experiment encompassing program installation and highest level power allowance. (a) Setup operation;

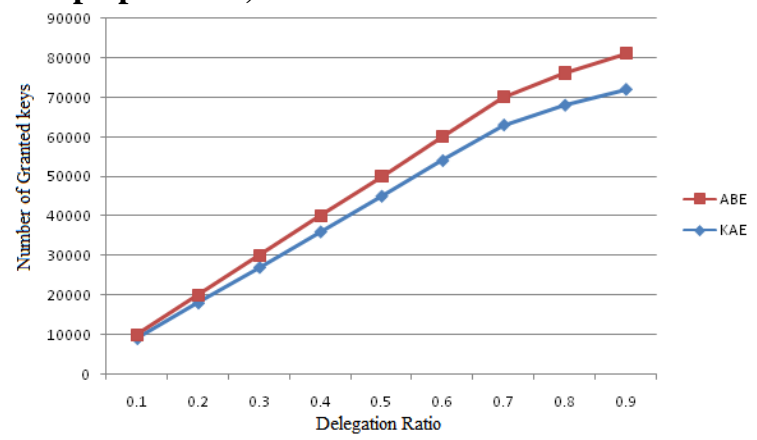

Figure 7: factors variance (na) which is desired for different methods in the condition such as 65536 sessions of data.

The implementation examples during the installation process the Key Gen will be ensuring the self-sustaining condition of the mission expense r. during the authentication process, Key Gen is needed three milliseconds and covering process needed 6:8 milliseconds. As predicted, the complication associated with working hour effects the yielding of outcome and Decrypt improves the designation price $r$ (which chooses the dimension the doled out set $\mathrm{S}$ ). The minute outcome also conform to what would be clear from the sum in Draw out and Decrypt process - two coupling capacities consumes insignificant hour, the occupied size of hour needed for the Decrypt is around double of the Draw out. Watch that our checks took care of as much as 65536 mixed bag of periods (which is additionally the burden component), and have to be sufficiently giant for exceptional-grained

Published By:

Blue Eyes Intelligence Engineering \& Sciences Publication

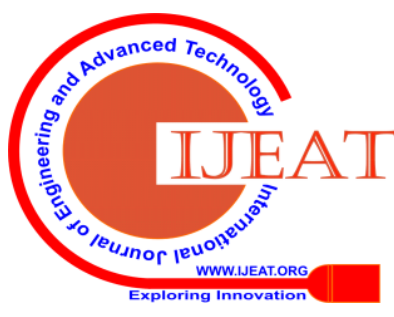


knowledge analyzing regularly [12]. In conclusion, we say that for initiatives where the mixed bag of determine content sessions is big yet the non-exclusive storage room is restrained, one have to mounted our systems utilize the sort-D coupling integrated with the PBC, which just desires one hundred seventy-bit to intend a factor in G. For $\mathrm{n}=216$, the assignment parameter wishes around 2:6 mb, which is as huge as a curb exceptional MP3 information document or a betterintention JPEG knowledge folder that a classic cellular telephone tin shop greater next various them. Be that as it may, we put away exorbitant relaxed storage room without the anxiety of taking good care of a structure of assignment session.

\section{CONCLUSION}

In the concluding note it could be mentioned that the $\mathrm{ABE}$ is acknowledging flexible and minute availability of the administration in thinking and planning process which effortlessly has a progressive framework structure of users by executing an assignment calculation to ABE. Therefore the ABE is not just encourages the substances credits because of flexible list of mixtures of capacities which also finishes prolific client crossing out on account of the feature activities of mechanisms. A set of enquiry could be generated during the development of the framework to protect the client's data. More computable resources such as cryptographic procedures are getting more flexible and on regular basis experts incorporate a few imperative elements for one and only program. In this discussion, researchers scrutinized the process of "pack" clues from the open clue cryptosystems. Whichever one more the strength set of grade, the agent tin directly acquire an total clue of constant measurement. Our methodology is more flexible than differed smooth clue undertaking which tin fair protect expance when ever each one clue talk about an indistinguishable arrangement of rights.

\section{REFERENCES}

1. Mohamed Nabeel, Elisa Bertino Fellow, "privacy keeping Delegated entry control in Public Clouds"," court cases in A preliminary variation of this paper appears within the lawsuits of the IEEE international convention on information Engineering(IRI '12)[1] as an invited paper.

2. M. Nabeel and E. Bertino, "privateers keeping delegated access manage in the storage as a provider mannequin," in EEE international conference on knowledge Reuse and Integration (IRI), 2012

3. N. Shang, M. Nabeel, F. Percent, and E. Bertino, "A privateerspreserving approach to policy-founded content material dissemination," in ICDE '10: lawsuits of the 2010 IEEE twenty sixth worldwide conference on data Engineering, 2010.

4. M. Nabeel, E. Bertino, M. Kantarcioglu, and B. M. Thuraisingham, "toward privateers maintaining access manage within the cloud," in proceedings of the seventh worldwide conference on Collaborative Computing: Networking", applications and Work sharing, ser. Collaborate Com '11, 2011, pp. 172-a hundred and eighty.

5. M.Nabeel, Noshing, and E.Bertino, "Privacy preserving policy based content sharing in public clouds," IEEE Transactions on Knowledge and Data Engineering, 2012.
6. M. Nabeel and E. Bertino, "Towards attribute based group key management," in Proceedings of the 18th ACM conference on Computer and communications security, Chicago, Illinois, USA, 2011.

7. M.Nabeel and E.Bertino, "Attribute based group key management," IEEE Transactions on Dependable and Secure Computing, 2012.

8. J.-M. Do, Y.-J. Song, and N. Park, "Attribute based proxy reencryption for data confidentiality in cloud computing environments," in Proceedings of the 1st International Conference on Computers, Networks, Systems and Industrial Engineering. Los Alamitos, CA, USA: IEEEComputerSociety,2011,pp.248-251.

9. Cheng-Kang Chu, Sherman S. M. Chow, Wen-Guey Tzeng, Jianying Zhou, and Robert H. Deng, "Key-Aggregate Cryptosystem for Scalable Data Sharing in Cloud Storage", proceedings in This work was supported by the Singapore A*STAR project SecDC-11217-2014.

10. S. S. M. Chow, Y. J. He, L. C. K. Hui, and S.-M. Yiu, "SPICE Simple Privacy-Preserving Identity-Management for Cloud Environment," in Applied Cryptography and Network Security ACNS 2012, ser. LNCS, vol. 7341. Springer, 2012, pp. 526-543.

11. L. Hardesty, "Secure computers aren't so secure," MIT press, 2009, http://www.physorg.com/news176107396.html.

12. C. Wang, S. S. M. Chow, Q. Wang, K. Ren, and W. Lou, "Privacy- Preserving Public Auditing for Secure Cloud Storage,' IEEE Trans. Computers, vol. 62, no. 2, pp. 362-375, 2013.

13. B. Wang, S. S. M. Chow, M. Li, and H. Li, "Storing Shared Data on the Cloud via Security-Mediator," in International Conference on Distributed Computing Systems - ICDCS 2013. IEEE, 2013.

14. S. S. M. Chow, C.-K. Chu, X. Huang, J. Zhou, and R. H. Deng, "Dynamic Secure Cloud Storage with Provenance," in Cryptography and Security: From Theory to Applications - Essays Dedicated to Jean-Jacques Quisquater on the Occasion of His 65th Birthday, ser.LNCS, vol. 6805. Springer, 2012, pp. 442-464.

\section{AUTHORS PROFILE}

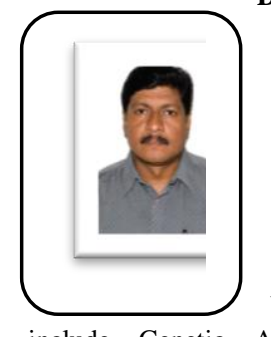

Dr.V.N.Rajavarman graduated in M.Sc Computer Science from Sri poondi pushpam college, Thanjavurand later completed his Masters Degree in ME Information Technology. He completed his Ph.D in Computer Science \& Engineering from Dr.MGR Educational \& Research Institute University in Feb 2011. Presently he is working as the Professor and Deputy Dean(Phase-II) of Dr.M.G.R Educational and Research Institute University, Chennai. His Research Interests include Genetic Algorithms, Data Mining and Warehousing, Cryptography and Cloud Computing

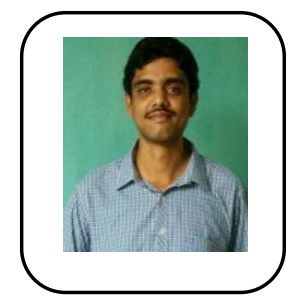

Mr.M.Srinivasarao is working as Assistan Professor in Computer Science and Engineering Department of PACE Institute of Technology and Sciences, Ongole. $\mathrm{He}$ is having 7 years, 5 months of rich Teaching Experience. His Research areas include Data Analytics, Data Mining, Machine Learning and Computer Networks 


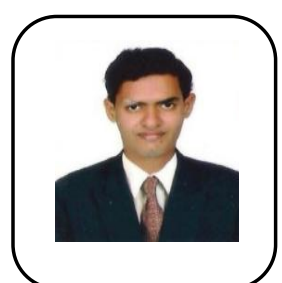

T.Venkata Satya Vivek completed his graduation under the Department of Computer Science \& Engineering from Vishnu Institute of Technology, Bhimavaram, India, and Post Graduation in the Specialization of Computer Networks and Security from KL University, Vijayawada. $\mathrm{He}$ is pursuing his $\mathrm{PhD}$ under the esteemed guidance of Dr.V.N Rajavarman (Professor \& Deputy Dean - Phase II) from Dr.M.G.R Educational and Research Institute University,Chennai. Presently he is working as Assistant Professor in VNR Vignana Jyothi Institute of Engineering and Technology, Hyderabad. His Research Interests includes Cryptography, Data Hiding in Images, Machine Learning. 